\title{
The clinical significance of CXCL5 in non-small cell lung cancer
}

This article was published in the following Dove Press journal:

OncoTargets and Therapy

Number of times this article has been viewed

Kongju $\mathrm{Wu}^{1, *}$
Shengnan $\mathrm{Yu}^{2, *}$
Qian Liu
Xianguang Bai'
Xinhua Zheng'
Kongming Wu ${ }^{2}$

'Medical School of Pingdingshan University, Pingdingshan, Henan, ${ }^{2}$ Department of Oncology, Tongji Hospital of Tongji Medical College, Huazhong University of Science and Technology, Wuhan, Hubei, People's Republic of China

*These authors contributed equally to this work
Correspondence: Kongming Wu Department of Oncology, Tongji Hospital of Tongji Medical College, Huazhong University of Science and Technology, 1095 Jiefang Avenue, Wuhan, Hubei 430030, People's Republic of China Email kmwu@tjh.tjmu.edu.cn

Xinhua Zheng

Medical School of Pingdingshan

University, Pingdingshan, Henan,

People's Republic of China

Email zhengxinhua70@I63.com
Abstract: As a CXC-type chemokine, ENA78/CXCL5 is an important attractant for granulocytes by binding to its receptor CXCR2. Recent studies proved that CXCL5/CXCR2 axis plays an oncogenic role in many human cancers. However, the exact clinical significance of CXCL5 in lung cancer has not been well defined. Here, we found that the serum protein expression of CXCL5 was significantly increased in non-small cell lung cancer (NSCLC) compared with that in healthy volunteers. Immunohistochemistry staining revealed that CXCL5 protein was higher in various lung cancer tissues compared with normal tissues. Moreover, CXCL5 expression correlated with histological grade, tumor size, and TNM stage in NSCLC. Elevated CXCL5 protein abundance predicted poor overall survival in adenocarcinoma patients. Further meta-analysis demonstrated that CXCL5 mRNA expression was also positively associated with tumor stage, lymph node metastasis, and worse survival. Kaplan-Meier plot analyses indicated high CXCL5 was associated with short overall survival and progression-free survival. Together, these results indicated that CXCL5 may be a potential biomarker for NSCLC.

Keywords: non-small cell lung cancer, chemokine, CXCL5, migration, prognosis

\section{Introduction}

Lung cancer is currently one of the world's most lethal diseases. Moreover, the incidence of lung cancer has continuously risen over the last 10 years in most countries. ${ }^{1}$ Histologically, lung cancer is divided into two categories: small cell carcinoma (SCC) and non-small cell carcinoma (NSCLC). NSCLC consists of several subtypes, including adenocarcinoma (ADC), squamous cell lung cancer (SQC), large cell lung cancer, and other rarer types. Although the discoveries of driver-mutations, such as EGFR and ALK tyrosine kinase inhibitors, and corresponding targeted therapies have dramatically improved the prognosis of a portion of NSCLC patients, the outcome for most lung cancer patients is still unsatisfactory due to late stage diagnosis. ${ }^{2}$ As the current staging system is imprecise for estimating prognosis of lung cancer, identification of "ready to use" molecular markers for early diagnosis, progression monitoring, and prognosis prediction is critical for precision and personal management. ${ }^{3}$

Through autocrine and paracrine signaling, cancer cells acquire growth signals and interact with the microenvironment, those cytokines and chemokines are potential biomarkers or therapeutic targets. ${ }^{4,5}$ In the search for putative prognostic markers for early stage NSCLC, Kwalczuk et al evaluated the mRNA level of a panel of chemokines using real-time PCR. Of 23 analyzed genes, only CXCL5 was found to statistically significantly influence both overall and disease-free survival. ${ }^{6}$ In association with its receptor, CXCR2, CXCL5 activates PI3K/AKT, MAP kinase, and $\beta$-catenin pathway to induce EMT and promote cancer cell migration and invasion. ${ }^{7-9}$ In addition 
to intracellular signaling transduction, CXCL5 induced myeloid-derived suppressor cell accumulation in a renal cell microenvironment leading to immune invasion. ${ }^{10}$ CXCL5 attracted $\mathrm{CXCR}^{+}$neutrophils into the tumor microenvironment to promote tumor progression, mediate resistance to therapy, and regulate immunosuppression. ${ }^{11}$

CXCL5 expression is regulated by oncogenes and tumor suppressors. An in vitro study demonstrated that gain of function mutant p53 activated the expression of CXCL5, whereas wild-type p53 repressed CXCL5 promoter activity, indicating a mechanistic link between mutant p53 proteins and chemokines in cell motility. ${ }^{12}$ FoxM1b is abundantly expressed in human NSCLC and transcriptionally induces expression of genes essential for cellular proliferation. A transgenic mouse model proved that FoxM1b increased expression of chemokine ligands CXCL5, CXCL1, and CCL3. ${ }^{13}$

The clinical value of CXCL5 in diagnosis and prognosis has been investigated in several cancers. Zhu et al reported that the sensitivity and specificity of CXCL5/creatinine ratio in predicting bladder cancer was 80.4 and $61.3 \%$, respectively. Increased CXCL5 expression in cancer tissue predicted poor survival in bladder cancer patients. Detection of CXCL5 expression in urine may be a non-invasive method for bladder cancer diagnosis. ${ }^{14}$ The high-CXCL5-expression group exhibited poor overall survival (OS) after curative hepatic resection and the presence of tumor-infiltrating neutrophils was associated with CXCL5 expression in tumor cells. ${ }^{15}$

So far, most published studies were from single institutions with relatively small patient samples, convincing results from large data analysis are still lacking. This study investigated the protein expression of CXCL5 in tumor tissue and peripheral serum of NSCLC patients. In combination with meta-analysis and exploration of an open big database, the association of CXCL5 with clinical-pathological parameters and prognosis was evaluated.

\section{Material and methods Human lung cancer tissue microarray (TMA)}

To evaluate the expression of CXCL5 in normal lung vs various lung tumor tissues, two commercially available human TMAs were used. LC1201 with core diameter of $1 \mathrm{~mm}$ was purchased from US Biomax (Rockville, MD, USA) containing 27 cases of ADCs (grade 1-3) and 27 squamous carcinomas (grade 1-3), eight papillary ADCs, eight SCCs, eight lung atypical carcinoids, eight ADCs with lepidic pattern (bronchioloalveolar carcinomas), two giant cell types, eight large cell carcinomas, 12 lung cancer metastases, six normal lung tissues from autopsy, and six normal adjacent lung tissues. HLug-Ade150Sur was purchased from Outdo Biotech containing 75 human lung ADC tissues with matched adjacent lung tissues with survival follow-up for 3-5 years.

\section{Immunohistochemical staining and quantification analysis}

Immunohistochemical staining was performed by Bios Biotech, Inc, with a 2-step protocol as previously described. ${ }^{16}$ CXCL5 antibody was purchased from Santa Cruz Biotechnology Inc. (Dallas, TX, USA) (sc-292642). For quantification, four fields at $200 \times$ magnification of each spot were selected for immunohistochemical scoring. Scores were calculated on intensity and proportion of positive tumor cells in the whole tissue stains according to the Fromowitz standard as described. ${ }^{17}$ The staining intensity was scored as 0 (no staining), 1 (weak staining, light yellow), 2 (moderate staining, yellow brown), and 3 (strong staining, brown). The proportions of stained tumor cells were classified as 1 ( $0 \%-25 \%$ positive cells $), 2$ (26\%-50\% positive cells), 2 (51\%-75\% positive cells), and $3(76 \%-100 \%$ positive cells). The multiplication for intensity and proportion was utilized to represent the protein levels of CXCL5.

\section{ELISA}

The blood samples were collected from approximately 100 patients who were diagnosed as having ADC at various stages by April 2015 in Tongji Hospital. All patients whose blood samples were used in this research provided written informed consent, and the study protocol was approved by the ethics committee of the Tongji Hospital of Huazhong University of Science and Technology. Serum samples from patients with obvious symptoms of inflammation were excluded. The ELISA kit for ENA78 (CXCL5) was purchased from CloudClone Corp (Katy, TX, USA). Diluted samples, including standard CXCL5 content, negative control and serums, were pipetted into 96-well plates pre-coated with anti-CXCL5. All procedures including incubation, washing, and detection were performed following standard manufacturer's protocols. After stopping the reaction, optical densities (ODs) were measured at $450 \mathrm{~nm}$ using a BioTek microplate reader. The intensity of this signal is directly proportional to the concentration of human CXCL5 present in the standard specimen, standard curve demonstrated the linearity $\mathrm{R}^{2}=0.9943$.

\section{Meta-analysis for CXCL5 on published Gene Expression Omnibus (GEO) databases}

We carried out a comprehensive search of relevant GEO databases for mRNA expression of CXCL5 through 
ArrayExpress and Oncomine as previously published. ${ }^{18}$ The datasets meeting the following criteria were included: 1) the datasets were about human NSCLC cancer; 2) CXCL5 mRNA expression was measured in these databases; 3) clinical characteristics of patients were shown in these databases; 4) the sample capacity was more than 50. Only the latest and most complete datasets were included when several databases shared common patients. At last, a total of 21 independent human NSCLC microarray databases with the mRNA expression of CXCL5 and clinical information were enrolled in this systematic analysis. Cutoff value for CXCL5 was median expression. OS was evaluated by Cox proportional HR and 95\% CI. HR was employed to assess the survival outcome of lung cancer patients with high mRNA expression of CXCL5 and HR $>1$ indicated that high expression of CXCL5 predicted worse survival of patients. Statistical analysis was performed based on the guidelines of Meta-Analysis of Observational Studies. The STATA software package (Stata Corp LP, College Station, TX, USA) was employed to perform the meta-analysis.

\section{Kaplan-Meier plotter}

Kaplan-Meier survival curves with HR and log-rank $P$-value were calculated and plotted with the analysis tool which can be accessed online at: http://kmplot.com/analysis. The background database, downloaded from GEO, offers gene expression data, relapse-free and OS information. The affymetrix ID for CXCL5 was 207852, which includes 2,437 cases of lung ADC and squamous cell carcinomas. The follow-up time threshold was 60 months. The patients were split by median expression of CXCL5. The KaplanMeier survival curves were downloaded from the website and resized in Adobe Illustrator CS5.

\section{Statistical analysis}

Statistical analyses between groups were calculated by Student's $t$-test and one-way ANOVA and the $P$-value was set at 0.05 . Univariate cumulative survival analyses for relapsefree survival and OS were calculated using the Kaplan-Meier method with the log-rank test. Statistical analyses were conducted using SPSS 19.0 and GraphPad Prism 5.0. All data were presented as the mean \pm standard error $(\mathrm{SE})$.

\section{Results}

\section{Increased expression of CXCL5 in various kinds of lung cancer}

The expression of CXCL5 in normal and lung cancer tissues was examined by immunohistochemical staining.
The representative images were shown in Figure 1A-I. The average score for normal lung tissue was $0.72 \pm 0.15$, all kinds of lung cancer tissues were $2 \sim 3$ folds higher than normal (Figure 1J). Small cell lung cancer (SCLC) demonstrated relatively lower CXCL5 than NSCLC. The average scores for ADC, squamous cell carcinoma, and papillary cancer were $2.48 \pm 0.21,2.51 \pm 0.23$, and $2.25 \pm 0.52$, respectively. Metastatic tissue showed higher CXCL5 than original tumor, but without reaching statistically significant difference.

\section{Expression of CXCL5 correlated with cancer progression and prognosis in lung ADC}

We analyzed the expression of CXCL5 in 75 cases of lung ADC with matched normal tissue and clinical-pathological parameters. The protein abundance of CXCL5 was positively correlated with tumor tissue grade, size, and TNM stage (Figure 2A-C). However, there was no statistical difference of CXCL5 expression between tumors with or without lymph node metastasis (Figure 2D). We investigated the association between cumulative OS and clinical-pathological parameters by Cox univariate regression analysis. Patients were divided into CXCL5 high and low subgroups based on the intensity of 3-4 vs $1-2$. The median OS time of the CXCL5 high and low groups were 48.866 \pm 2.344 and $35.419 \pm 4.566$, respectively ( $P$-value 0.035 ) (Figure $3 \mathrm{~A})$. However, Cox multi-various analysis including sex, age, grade, CXCL5 expression, lymph node, and TNM stage demonstrated that only T1 -2 vs T3-4 had statistical significance $(P=0.005)$ (Figure 3B).

\section{Elevated serum CXCL5 in lung ADC}

Next, we examined the serum CXCL5 abundance with a commercial ELISA kit. The standard curve between CXCL5 amount and OD value demonstrated an excellent linear relationship with $\mathrm{R}^{2}=0.9943$ (Figure 4A). Fresh serum was isolated from 20 normal samples and 48 ADC patients covering pathological stage I-IV and distinct histological grades. Of 48 ADC patients, 23 were operable, stage I-IIIa, while the rest of the patients were stage IIIb-IV. The results showed that ADC patients had more than a 3-fold increase of serum CXCL5 compared to normal samples (T/Normal, $P<0.0001$ ) (Figure 4B). The serum CXCL5 was higher in patients with T3-4 tumors than those with T1-2 tumors, however the difference just missed statistical significance $(P=0.056)$. Because of the limited sample number, we did not observe a remarkable difference of CXCL5 expression in relation to tumor grade. 

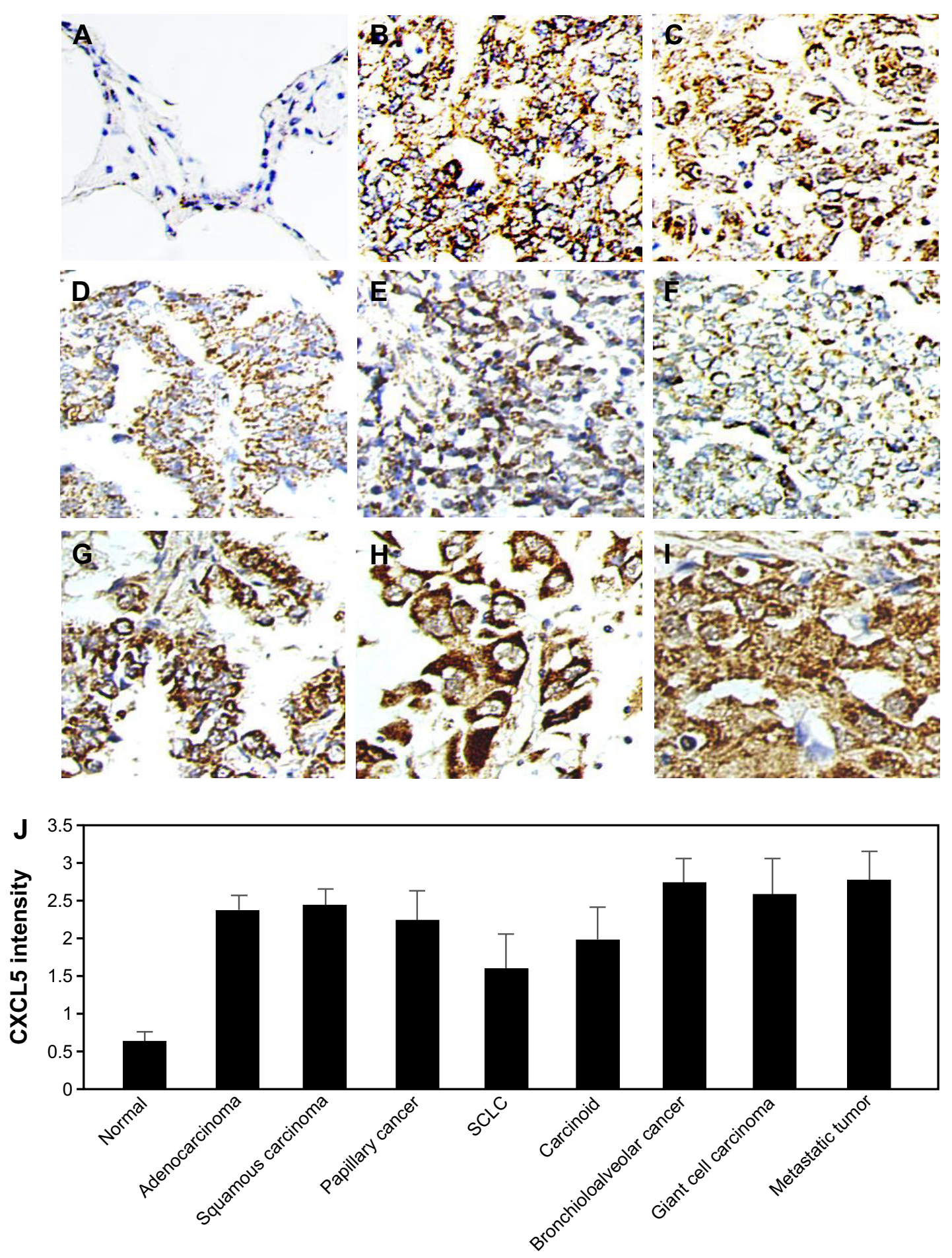

Figure I Representative images of CXCL5 expression by immunohistochemical staining.

Notes: (A) Normal, (B) adenocarcinoma, (C) squamous carcinoma, (D) papillary cancer, (E) SCLC, (F) carcinoid, (G) bronchioloalveolar cancer, (H) giant cell carcinoma, (I) metastatic tumor, (J) quantitative score of CXCL5. Magnification 200X.

Abbreviation: SCLC, small cell lung cancer.

Meta-analysis of CXCL5 mRNA expression with clinical-pathological characteristics

Nine datasets met the selection standard and were used to perform pooled analysis to evaluate the HR of CXCL5 expression. The patients were divided into CXCL5 high and low subgroups based on the median CXCL5 mRNA value. High expression of CXCL5 correlated with short OS in NSCLC (pooled HR $=1.2,95 \%$ CI, 1.03-1.39, $P=0.971$, and $I^{2}=0.0 \%$ ) (Figure 5A). Subgroup analysis indicated that 
A

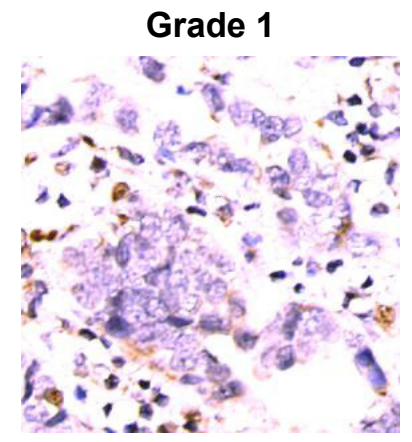

B

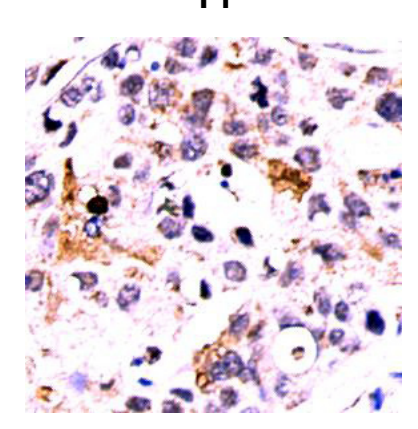

C

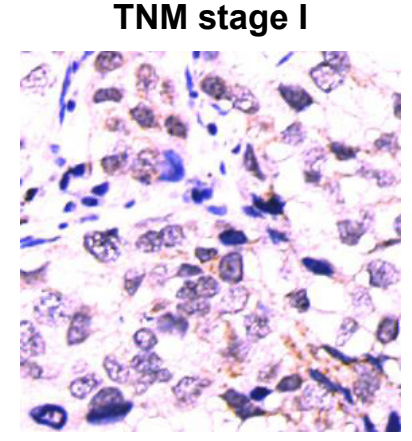

D

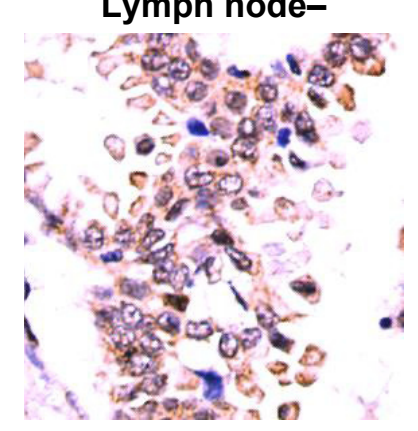

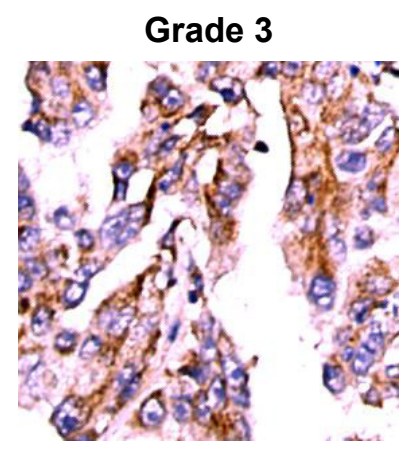

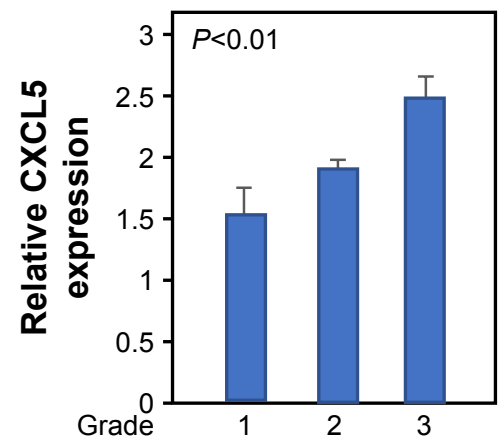

T3
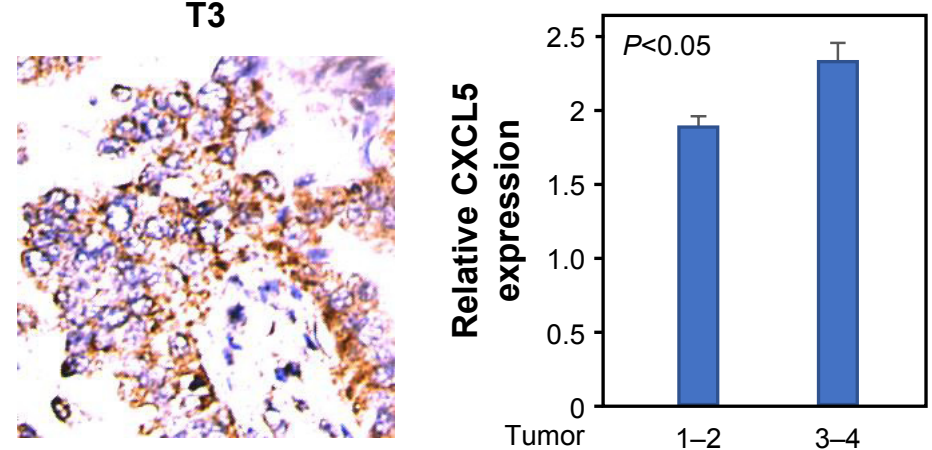

TNM stage III
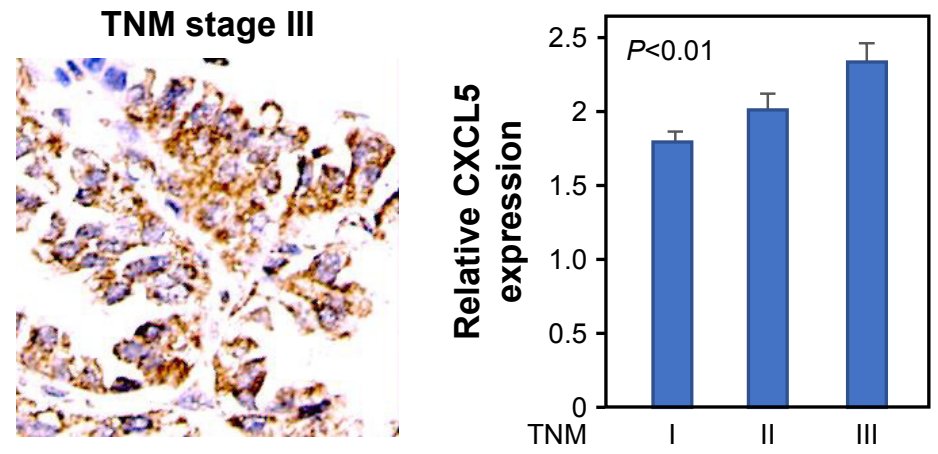

Figure 2 Association of CXCL5 expression with pathological parameters in adenocarcinoma by immunohistochemical staining.

Notes: (A) Grade I vs 3, (B) T stage I vs 3, (C) TNM stage I vs III, (D) lymph node positive vs negative. Left panel: representative images, right panel: quantitative score.

major risk was derived from $\mathrm{ADC}$, however, the $P$-value failed to reach statistical significance.

Thirteen gene expression datasets were extracted to compare the mRNA level of CXCL5 between ADC and squamous cell carcinoma. The results showed that the CXCL5 mRNA profile was dramatically higher in ADC tumors than squamous carcinomas with OR 1.24 (95\% CI: 1.01-1.53) (Figure 5B). Tumor tissues were grouped into early TNM stage I-II or late stage III-IV for all NSCLC patients, CXCL5 expressions were higher in III-IV than I-II with OR 1.27 (95\% CI: 1.01-1.58) (Figure 5C). Then, subgroup analysis was performed to evaluate $\mathrm{ADC}$ and 
A

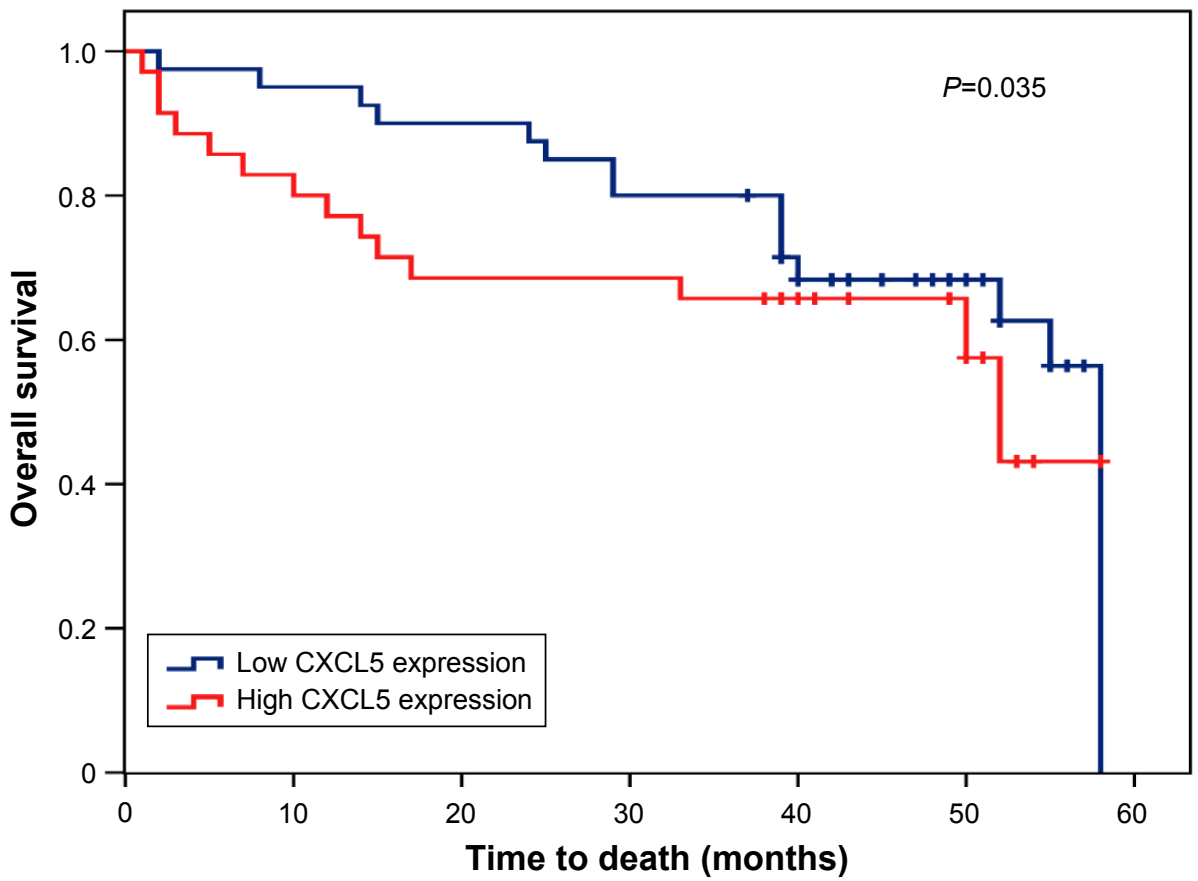

\begin{tabular}{|c|c|c|c|c|c|c|c|c|}
\hline & B & SE & Wald & $d f$ & Sig. & $\operatorname{Exp}(B)$ & \multicolumn{2}{|c|}{$95 \% \mathrm{CI}$} \\
\hline Sex & 0.480 & 0.421 & 1.302 & 1 & 0.254 & 1.617 & 0.708 & 3.689 \\
\hline Age & 0.005 & 0.022 & 0.050 & 1 & 0.822 & 1.005 & 0.963 & 1.049 \\
\hline Grade & 0.225 & 0.638 & 0.124 & 1 & 0.725 & 1.252 & 0.358 & 4.376 \\
\hline CXCL5 & -0.215 & 0.439 & 0.240 & 1 & 0.624 & 0.806 & 0.341 & 1.906 \\
\hline $\begin{array}{l}\text { T1-2 vs } \\
\text { T3-4 }\end{array}$ & -1.238 & 0.443 & 7.829 & 1 & 0.005 & 0.290 & 0.122 & 0.690 \\
\hline Lymph & -0.476 & 0.526 & 0.819 & 1 & 0.365 & 0.621 & 0.222 & 1.741 \\
\hline
\end{tabular}

Figure 3 Prognostic value of CXCL5 in adenocarcinoma.

Notes: (A) Kaplan-Meier curve of patients with low or high CXCL5 expression. Median level was used to divide patients into subgroups. (B) Multivariate analysis of clinical and pathological parameters.

Abbreviations: Sig, significance; Exp, odd ratio value; B, beta; SE, standard error.

squamous cell carcinoma, the two major subtypes of NSCLC. The collected data demonstrated that increased CXCL5 expression was associated with advanced stage only in ADC with OR 1.39 (95\% CI: 1.01-1.88) (Figure 5D), but not in SQC. Further analysis of CXCL5 with lymph node status indicated that tumor tissues with high CXCL5 expression had increased risk for lymph node metastasis (OR 1.37, 95\% CI: 1.06-1.77) (Figure 5E). Similarly, subgroup analysis showed this association only existed in ADC with OR 1.43
(95\% CI: 1.06-1.93) (Figure 5F). However, no association was observed between the CXCL5 expressions and tumor tissue differentiation.

\section{Increased CXCL5 expression predicts poor survival in NSCLC}

CXCL5 mRNA expression profiles and follow-up data from 2,437 NSCLC patients were openly accessible from kmplot.com. The Kaplan-Meier curves from Cox univariate 
A

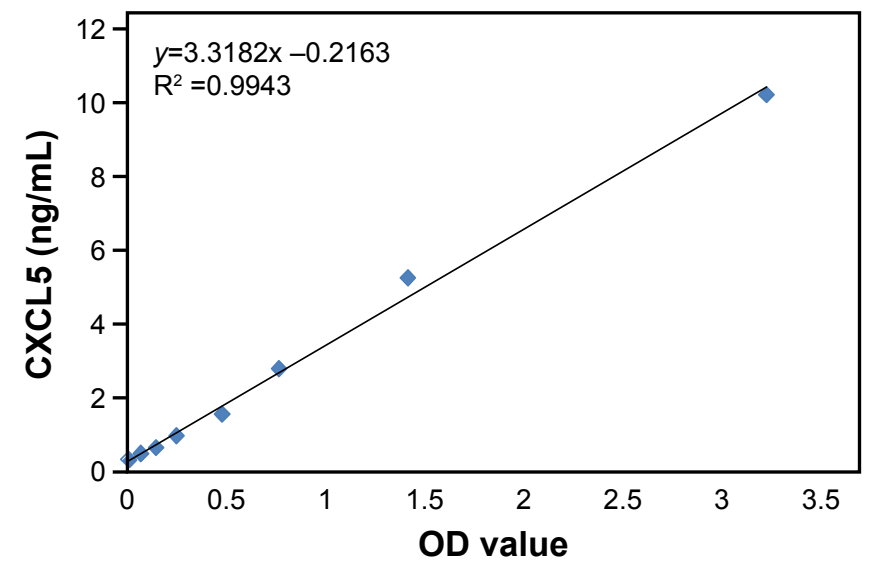

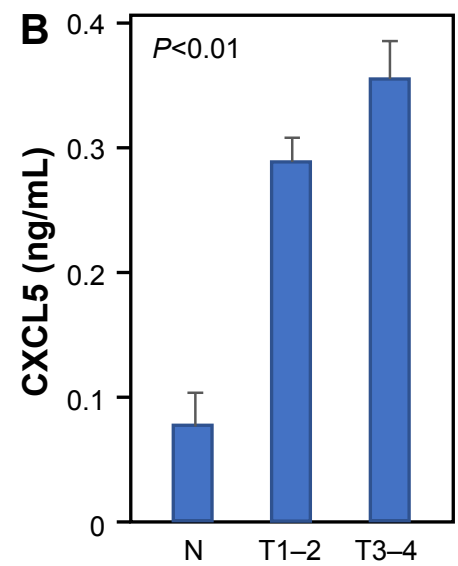

Figure 4 Serum CXCL5 concentration detected by ELISA.

Notes: (A) Standard curve of CXCL5 with OD value. (B) Peripheral serum CXCL5 in healthy volunteers and lung adenocarcinoma patients. Abbreviations: OD, optical density; $\mathrm{N}$, normal.

regression analysis indicated that patients with higher than median mRNA level of CXCL5 had shorter OS time (HR $1.16,95 \%$ CI 1.01-1.33, $P=0.036$ ) (Figure 6A), post-progression survival (PPS) time (HR 1.49, 95\% CI 1.15-1.93, $P=0.0024$ ) (Figure 6B), and progression-free survival (PFS) (HR 1.25, 95\% CI 1.03-1.52, $P=0.025$ ) (Figure 6C). Further subgroup analysis demonstrated that PFS in patients with lung ADC had marginal significance $(P=0.055)$ (Figure 6D), all other stratifications did not reach statistical significance, such as the CXCL5 expression with OS and PPS in ADC or squamous cell carcinoma. Together, these analyses indicated that high expression of CXCL5 correlated with unfavorable prognosis in patients with NSCLC.

\section{Discussion}

Besides well-known driver genes, many factors contribute to lung cancer pathogenesis and progression. ${ }^{40,41}$ Various biomarkers have been explored for diagnosis, targeted therapy, or prognosis. ${ }^{42-45}$ CXCL5 is a member of a proangiogenic subgroup of the CXC-type chemokine family. Emerging evidence has demonstrated that CXCL5 is involved in carcinogenesis and cancer progression. Arenberg et al first reported that CXCL5 was elevated in fresh NSCLC tissues and correlated with vascular density. In an in vivo tumor model, expression of CXCL5 promoted tumor growth. This function was blocked by neutralizing anti-CXCL5 antibodies accompanied with reduced tumor vascularity and spontaneous metastases. ${ }^{46}$ In human NSCLC, Cox-2-enhanced tumor growth was inhibited by neutralizing CXCL5 antibody, suggesting that CXCL5 was a key downstream target. ${ }^{47}$ CXCR2 expression in tumor cells was associated with smoking and poor prognosis. Analysis of gene expression profiles on
CXCR2 axis in human NSCLC cell lines and lung ADCs defined a gene cluster driven by CXCL5. Targeting CXCR2 axis may benefit patients with smoking-related lung ADC..$^{48}$ Similarly, our study showed that CXCL5 protein abundance in various types of lung cancer was 3 5-fold higher than in normal lung epithelial tissues. Specifically, CXCL5 in ADC tissue correlated with tissue differentiation status, tumor size, and TNM stage. Moreover, patients with high CXCL5 expression in ADC had short cumulative survival.

Surgical resection is still regarded as the standard therapy for early NSCLC, however, many tumors recurred in less than 5 years. In the search for circulating markers for lung cancer, Shiels et al evaluated 72 serum proteins using luminex bead-based assay and found that eleven were statistically significantly associated with lung cancer risk, including CXCL5 concentration with the OR approximately 3-6. ${ }^{49}$ Spaks measured peripheral concentration of CXCL1 and CXCL4 to CXCL12 with annual follow-up for 6 years. Results indicated that the average CXCL5 was $882.74 \mathrm{pg} / \mathrm{mL}$ for all 56 patients, $658.33 \mathrm{pg} / \mathrm{mL}$ for 30 patients without recurrence, and $1,213.33 \mathrm{pg} / \mathrm{mL}$ for 24 cases of local relapse or systemic cancer metastasis. This research suggested that monitoring inflammation and chemokines might identify high-risk patients for precise post-surgical management. ${ }^{50} \mathrm{In}$ concurrence with this report, our result showed that serum CXCL5 was 4.5-fold higher than in healthy volunteers and positively correlated with tumor stage. Meta-analysis of 20 sets of expression database indicated that high level of CXCL5 mRNA expression increased risk of death, at the same time, the Kaplan-Meier curve demonstrated that high level of CXCL5 expression decreased OS, PPS, and PFS in NSCLC. While pooled analysis of CXCL5 mRNA expression 


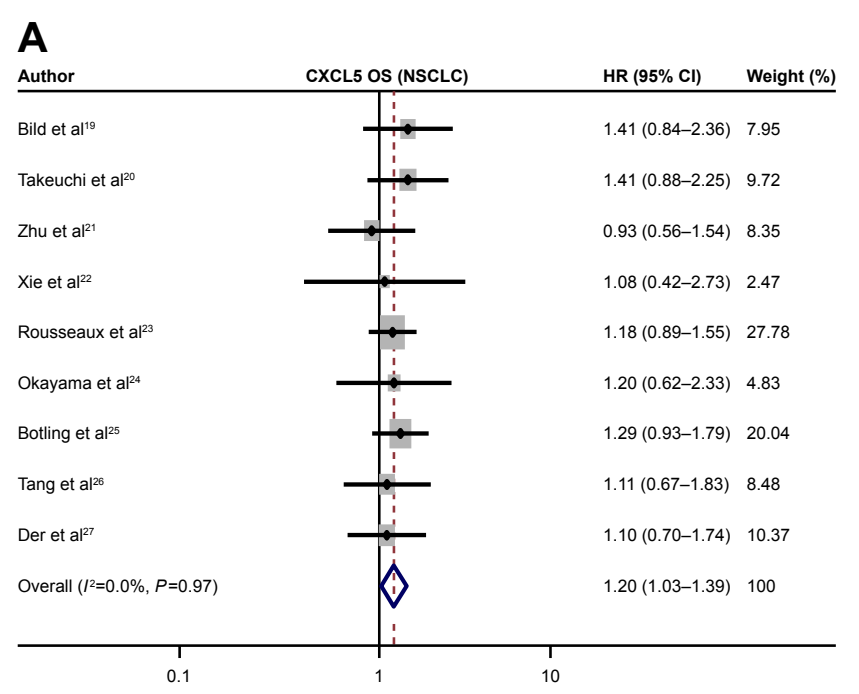

B

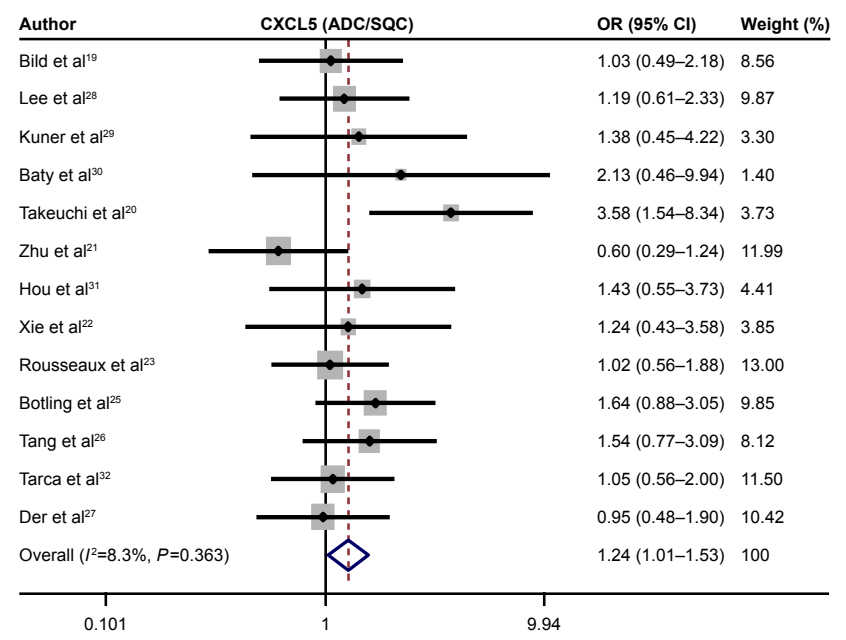

C

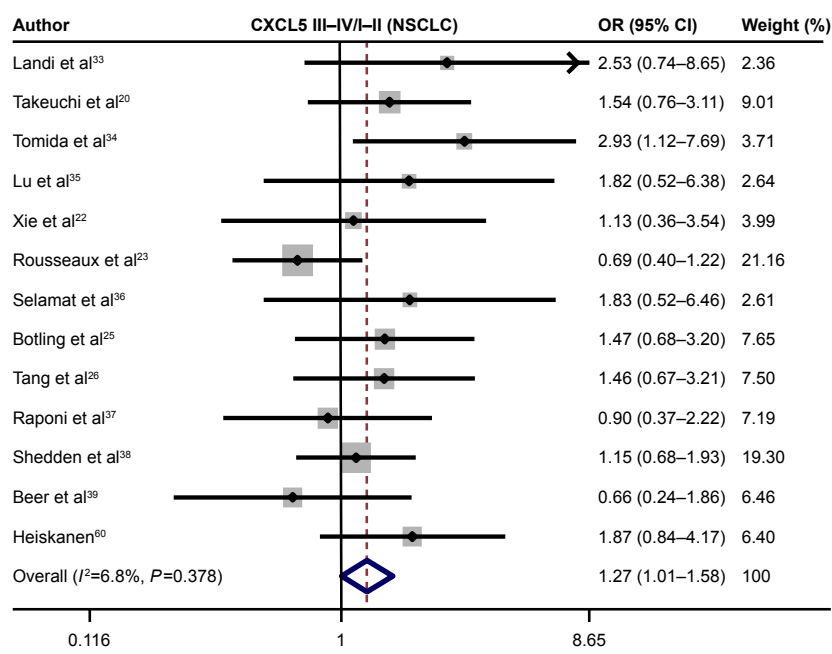

D

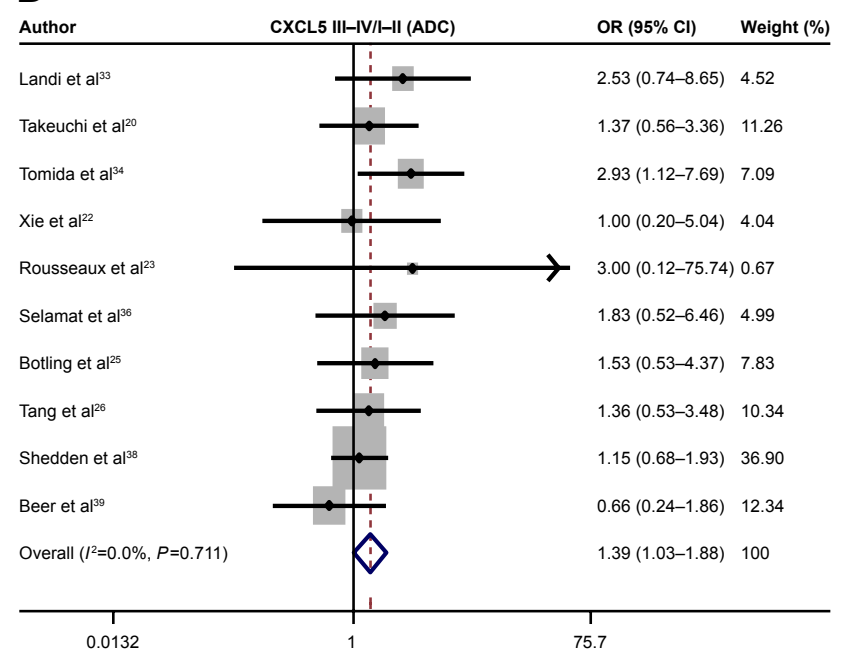

$F$

E

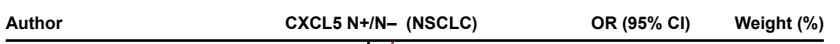

Baty et al ${ }^{30}$

Takeuchi et al $\mathrm{a}^{2}$

Der et $\mathrm{al}^{27}$

Tomida et al ${ }^{34}$

Shedden et al ${ }^{38}$

Raponi et a ${ }^{37}$

Overall $\left(I^{2}=10.6 \%, P=0.348\right)$

0.0685

OR $(95 \%$ CI) Weight (\%)

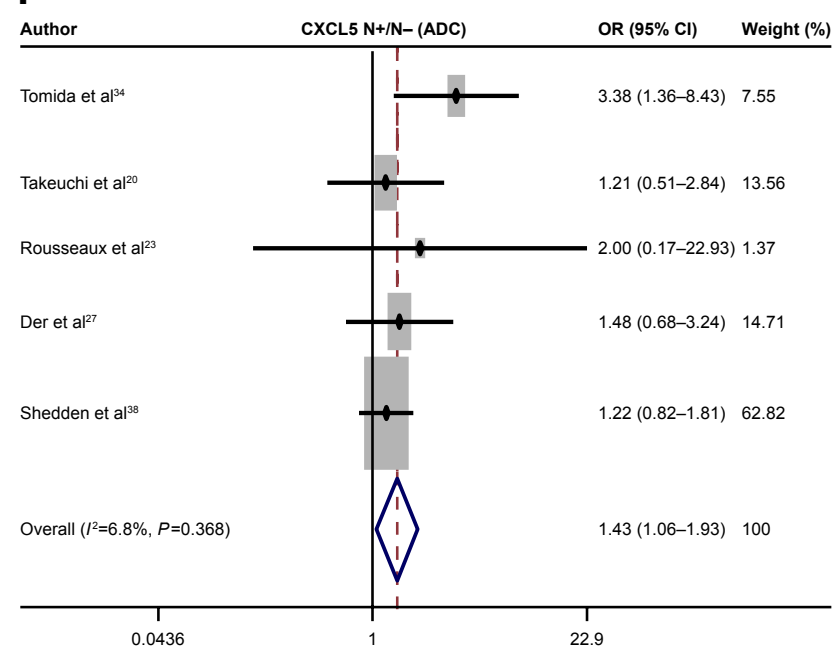

Figure 5 Forest plot of HR and OS of CXCL5.

Notes: (A) Relative risk of CXCL5 mRNA expression to OS in NSCLC. OR value of CXCL5 in ADC vs SQC (B) stages III-IV vs I-II in NSCLC (C) and ADC (D), OR value of CXCL5 comparing $\mathrm{N}+$ and $\mathrm{N}$ - patients in NSCLC (E) and ADC (F).

Abbreviations: HR, hazard ratio; OR, odds ratio; OS, overall survival; NSCLC, non-small cell lung cancer; ADC, adenocarcinoma; SQC, squamous cell lung cancer ; N+, lymph node positive; $\mathrm{N}-$, lymph node negative. 


\section{A}

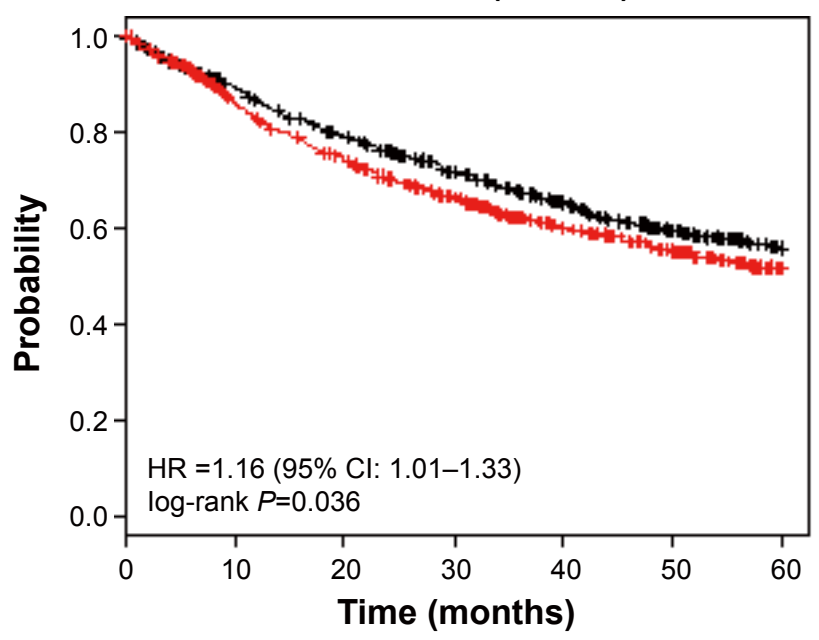

C

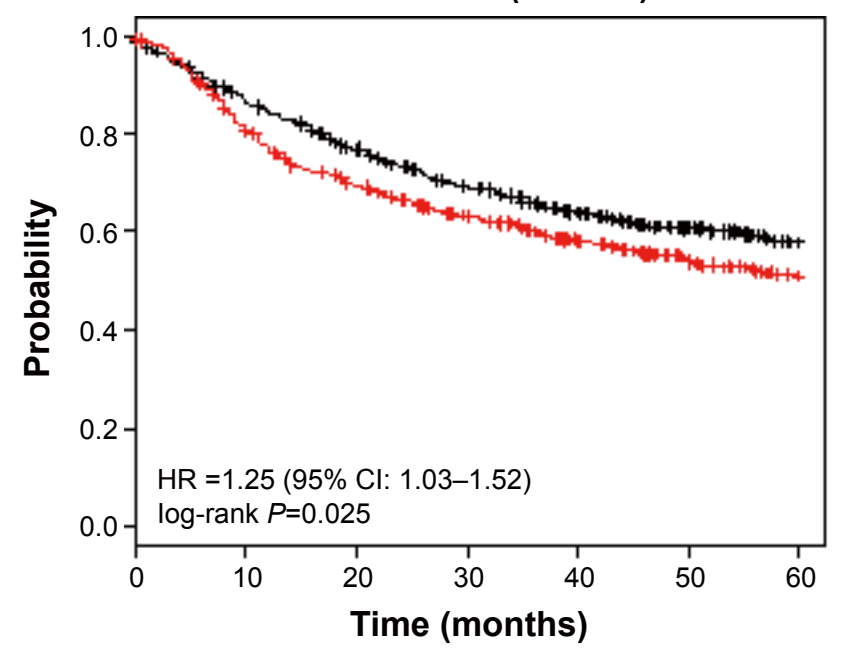

B CXCL5 PPS (NSCLC)

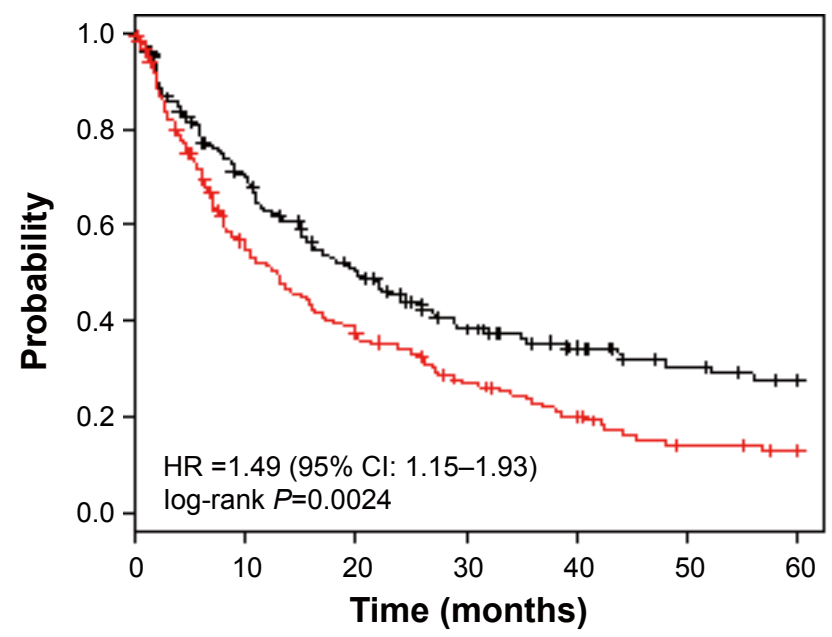

D CXCL5 PFS (ADC)

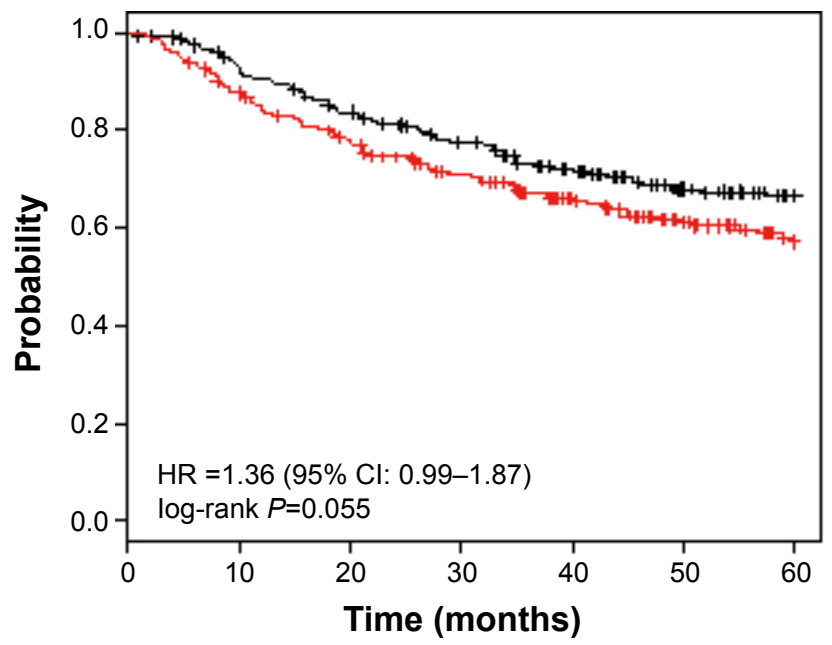

Expression

Low L High

Figure 6 Kaplan-Meier survival curve.

Notes: Association between CXCL5 and OS (A), PPS (B), and PFS (C) in NSCLC patients. (D) Association of CXCL5 with PFS in patients with ADC.

Abbreviations: HR, hazard ratio; OS, overall survival; PPS, post-progression survival; PFS, progression-free survival; ADC, adenocarcinoma; NSCLC, non-small cell lung cancer.

indicated positive association with lymph node metastasis, CXCL5 protein abundance in ADC did not show a statistical difference between the lymph node positive and negative subgroups. We attributed this seemingly contradictory result to the limited sample size and less precise measurement in immunohistochemical staining.

It is well-known that CXCL5 recruits neutrophils into the tumor microenvironment. ${ }^{11}$ To test whether neutrophil infiltration could be a surrogate marker for CXCL5, we performed a regression analysis between CXCL5 with neutrophil surface marker CD15. In several datasets, there was a statistically significant positive relationship, indicating that tumor tissues with high CXCL5 expression might host more inflammatory cells (Figure S1), supporting the previous study ${ }^{15}$ However, the correlation factor was not high enough to assume that this marker could replace CXCL5.

Whole-genome and transcriptome sequencing of tumor samples identified DACH1 as being involved in NSCLC. We previously reported that expression of DACH1 was significantly decreased in human NSCLC tissues and high DACH1 was a protection factor for OS and PFS, while expression of CXCL5 was increased and predicated poor prognosis. Further 
analysis demonstrated that DACH1 abundance was inversely correlated with CXCL5 in lung cancer cell lines and tumor tissues. ${ }^{51}$ The prognostic value of CXCL5 was also identified in other kinds of cancer. For example, high expression of both CXCR2 and CXCL5 in hepatocellular carcinoma showed a significant correlation with the activation of PI3K/Akt/ GSK-3 $\beta /$ Snail signaling and EMT phenotype, predicting poor prognosis. It may serve as a potential therapeutic target. ${ }^{52}$ Serum CXCL5 and SDF-1/CXCL12 levels were significantly higher in advanced gastric cancer groups than early gastric cancer group, high-risk and normal groups. Clinicalpathological serum CXCL5 was correlated with T-stage and distant metastasis. ROC curve and logistic regression demonstrated that serum CXCL5 and SDF-1/CXCL12 showed higher diagnostic accuracy compared with carcinoembryonic antigen in predicting gastric cancer. A combination of serum CXCL5, SDF-1/CXCL12, and CEA achieved 92.8\% specificity and $75.0 \%$ sensitivity to predict distant metastasis of gastric cancer. ${ }^{53}$ Zhang et al used a novel statistical model to test serum CXCL5 for prognosis in nasopharyngeal carcinoma patients. The serum CXCL5 cutoff point was $0.805 \mathrm{ng} / \mathrm{mL}$. Histology, T classification, clinical classification, and local recurrence were not associated with serum CXCL5 levels. However, serum CXCL5 levels were positively associated with lymph node classification, distant metastasis, and disease progression $(P<0.05)$. A high serum CXCL5 level predicted poor 6-year OS and PFS. Furthermore, this novel model successfully divided the patients into four risk subgroups. The $\mathrm{C}$-indices were 0.751 and 0.762 for the training set and the testing set, respectively. The novel statistical $\mathrm{C}-\mathrm{C}$ model combining serum CXCL5 levels and clinical classification could be helpful in predicting the prognosis of nasopharyngeal carcinoma patients. ${ }^{54}$ Since CXCL5 has cross-action with other pathways to promote tumor growth, interfering with CXCL5 might have a synergistic anti-tumor effect. For example, Snail promotes invasion and metastasis of NSCLC by inducing epithelial-mesenchymal transition. Overexpression of Snail in NSCLC led to increased angiogenesis accompanied by increased CXCL8 and CXCL5 concentrations in tumor homogenates. Furthermore, the tumorigenic action of Snail was abrogated with CXCR2 blockade, proving that CXCL5-CXCR2 axis mediated the oncogenic role of Snail. ${ }^{55}$ CXCL5 was highly expressed in androgen-independent prostate cancers and responsible for cell migration and epithelial-to-mesenchymal transition. In androgen-independent PC-3 and DU145 cells, CXCL5 increased Raf/MEK/ERK activation and Egr-1 to induce Snail expression. Inhibition of CXCL5-mediated ERK/
Egr-1/Snail signaling is an attractive therapeutic target for androgen-independent prostate cancer. ${ }^{56}$ As CXCL5 is an important mediator of tumor-derived angiogenesis, theoretically, blockade of either CXCL5 or CXCR2 may be a critical adjunct antiangiogenic therapy against pancreatic cancer. ${ }^{57}$

\section{Conclusion}

CXCL5 activated AKT, MAPK signal to promote tumor proliferation, invasion, and tumor growth. It recruits neutrophils through CXCR2 receptors and is involved in the recruitment and activation of neutrophils. Experimental evidence has proven that targeting this axis by neutralized antibody to CXCL5 or using chemical inhibitor to CXCR2 has anti-tumor effect. ${ }^{58}$ However, those experimental studies have not been transferred to targeted therapy. ${ }^{59}$ Our study found that high level of CXCL5 in the peripheral serum of ADC and protein abundance of CXCL5 in the tumor tissue correlated with aggressive characteristics and associated with poor OS. Meta-analysis supported the fact that enhanced CXCL5 mRNA expression and associated aggressive phenotypes were majorly linked to lung ADC. In summary, CXCL5 could be a potential screening and prognostic marker for lung ADC.

\section{Acknowledgments}

This study was supported by National Natural Science Foundation of China (grant no 81572608) and Wuhan Science and Technology Bureau (grant no 2017060201010170).

\section{Author contributions}

Kongju Wu and Shengnan Yu collected and analyzed the data and drafted some sections. Qian Liu participated in data collection, immunohistochemical analysis, and drafted some sections. Xianguang Bai collected data, performed some statistical analysis, and drafted some sections. Kongming Wu and Xinhua Zheng designed the study, evaluated results, and revised the manuscript. All authors contributed toward data analysis, drafting and revising the paper and agree to be accountable for all aspects of the work.

\section{Disclosure}

The authors report no conflicts of interest in this work.

\section{References}

1. Siegel RL, Miller KD, Jemal A. Cancer statistics, 2015. CA Cancer J Clin. 2015;65(1):5-29.

2. Dholaria B, Hammond W, Shreders A, Lou Y. Emerging therapeutic agents for lung cancer. J Hematol Oncol. 2016;9(1):138.

3. Wei S, Wang K. Long noncoding RNAs: pivotal regulators in acute myeloid leukemia. Exp Hematol Oncol. 2016;5:30. 
4. Liu Q, Yu S, Li A, Xu H, Han X, Wu K. Targeting interlukin-6 to relieve immunosuppression in tumor microenvironment. Tumour Biol. 2017; 39(6): 1010428317712445.

5. Liu Q, Li A, Tian Y, et al. The CXCL8-CXCR1/2 pathways in cancer. Cytokine Growth Factor Rev. 2016;31:61-71.

6. Kowalczuk O, Burzykowski T, Niklinska WE, Kozlowski M, Chyczewski L, Niklinski J. CXCL5 as a potential novel prognostic factor in early stage non-small cell lung cancer: results of a study of expression levels of 23 genes. Tumour Biol. 2014;35(5):4619-4628.

7. Gao Y, Guan Z, Chen J, et al. CXCL5/CXCR2 axis promotes bladder cancer cell migration and invasion by activating PI3K/AKT-induced upregulation of MMP2/MMP9. Int J Oncol. 2015;47(2):690-700.

8. Zhao J, Ou B, Han D, et al. Tumor-derived CXCL5 promotes human colorectal cancer metastasis through activation of the ERK/Elk-1/ Snail and AKT/GSK3ß/ $\beta$-catenin pathways. Mol Cancer. 2017; 16(1):70.

9. Hsu YL, Hou MF, Kuo PL, Huang YF, Tsai EM. Breast tumorassociated osteoblast-derived CXCL5 increases cancer progression by ERK/MSK1/Elk-1/snail signaling pathway. Oncogene. 2013;32(37) 4436-4447.

10. Najjar YG, Rayman P, Jia X, et al. Myeloid-derived suppressor cell subset accumulation in renal cell carcinoma parenchyma is associated with intratumoral expression of IL1 $\beta$, IL8, CXCL5, and Mip-1 $\alpha$. Clin Cancer Res. 2017;23(9):2346-2355.

11. Zhou SL, Dai Z, Zhou ZJ, et al. Overexpression of CXCL5 mediates neutrophil infiltration and indicates poor prognosis for hepatocellular carcinoma. Hepatology. 2012;56(6):2242-2254.

12. Yeudall WA, Vaughan CA, Miyazaki H, et al. Gain-of-function mutant p53 upregulates $\mathrm{CXC}$ chemokines and enhances cell migration. Carcinogenesis. 2012;33(2):442-451.

13. Wang IC, Meliton L, Tretiakova M, Costa RH, Kalinichenko VV, Kalin TV. Transgenic expression of the forkhead box M1 transcription factor induces formation of lung tumors. Oncogene. 2008;27(30):4137-4149.

14. Zhu X, Qiao Y, Liu W, et al. CXCL5 is a potential diagnostic and prognostic marker for bladder cancer patients. Tumor Biol. 2016;37(4): 4569-4577.

15. Okabe H, Beppu T, Ueda M, et al. Identification of CXCL5/ENA-78 as a factor involved in the interaction between cholangiocarcinoma cells and cancer-associated fibroblasts. Int J Cancer. 2012;131(10) 2234-2241.

16. Xu H, Wu KJ, Tian Y, et al. CD44 correlates with clinicopathological characteristics and is upregulated by EGFR in breast cancer. Int J Oncol. 2016;49(4):1343-1350.

17. Xu H, Yu S, Yuan X, Xiong J, Kuang D, Pestell RG, Wu K. DACH1 suppresses breast cancer as a negative regulator of CD44. Sci Rep. 2017; 7(1):4361.

18. Xu H, Wu KJ, Tian Y, et al. Expression profile of SIX family members correlates with clinic-pathological feature and prognosis of breast cancer: a systematic review and meta-analysis. Medicine (Baltimore) 2016;95(27):e4085.

19. Bild AH, Yao G, Chang JT, et al. Oncogenic pathway signatures in human cancers as a guide to targeted therapies. Nature. 2006; 439(7074):353-357.

20. Takeuchi T, Tomida S, Yatabe Y, et al. Expression profile-defined classification of lung adenocarcinoma shows close relationship with underlying major genetic changes and clinicopathologic behaviors. J Clin Oncol. 2006;24(11):1679-1688.

21. Zhu CQ, Ding K, Strumpf D, et al. Prognostic and predictive gene signature for adjuvant chemotherapy in resected non-small-cell lung cancer. J Clin Oncol. 2010;28(29):4417-4424.

22. Xie Y, Xiao G, Coombes KR, et al. Robust gene expression signature from formalin-fixed paraffin-embedded samples predicts prognosis of non-small-cell lung cancer patients. Clin Cancer Res. 2011;17(17): 5705-5714.

23. Rousseaux S, Debernardi A, Jacquiau B, et al. Ectopic activation of germline and placental genes identifies aggressive metastasis-prone lung cancers. Sci Transl Med. 2013;5(186):186ra66.
24. Okayama H, Kohno T, Ishii Y, et al. Identification of genes upregulated in ALK-positive and EGFR/KRAS/ALK-negative lung adenocarcinomas. Cancer Res. 2012;72(1):100-111.

25. Botling J, Edlund K, Lohr M, et al. Biomarker discovery in non-small cell lung cancer: integrating gene expression profiling, meta-analysis, and tissue microarray validation. Clin Cancer Res. 2013;19(1):194-204.

26. Tang H, Xiao G, Behrens C, et al. A 12-gene set predicts survival benefits from adjuvant chemotherapy in non-small cell lung cancer patients. Clin Cancer Res. 2013;19(6):1577-1586.

27. Der SD, Sykes J, Pintilie M, et al. Validation of a histology-independent prognostic gene signature for early-stage, non-small-cell lung cancer including stage IA patients. J Thorac Oncol. 2014;9(1):59-64.

28. Lee ES, Son DS, Kim SH, et al. Prediction of recurrence-free survival in postoperative non-small cell lung cancer patients by using an integrated model of clinical information and gene expression. Clin Cancer Res. 2008;14(22):7397-7404.

29. Kuner R, Muley T, Meister M, et al. Global gene expression analysis reveals specific patterns of cell junctions in non-small cell lung cancer subtypes. Lung Cancer. 2009;63(1):32-38.

30. Baty F, Facompré M, Kaiser S, et al. Gene profiling of clinical routine biopsies and prediction of survival in non-small cell lung cancer. $\mathrm{Am}$ J Respir Crit Care Med. 2010;181(2):181-188.

31. Hou J, Aerts J, den Hamer B, et al. Gene expression-based classification of non-small cell lung carcinomas and survival prediction. PLoS One. 2010;5(4):e10312.

32. Tarca AL, Lauria M, Unger M, et al. Strengths and limitations of microarraybased phenotype prediction: lessons learned from the IMPROVER Diagnostic Signature Challenge. Bioinformatics. 2013;29(22):2892-2899.

33. Landi MT, Dracheva T, Rotunno M, et al. Gene expression signature of cigarette smoking and its role in lung adenocarcinoma development and survival. PLoS One. 2008;3(2):e1651.

34. Tomida S, Takeuchi T, Shimada Y, et al. Relapse-related molecular signature in lung adenocarcinomas identifies patients with dismal prognosis. J Clin Oncol. 2009;27(17):2793-2799.

35. Lu TP, Tsai MH, Lee JM, et al. Identification of a novel biomarker, SEMA5A, for non-small cell lung carcinoma in nonsmoking women. Cancer Epidemiol Biomarkers Prev. 2010;19(10):2590-2597.

36. Selamat SA, Chung BS, Girard L, et al. Genome-scale analysis of DNA methylation in lung adenocarcinoma and integration with mRNA expression. Genome Res. 2012;22(7):1197-1211.

37. Raponi M, Zhang Y, Yu J, et al. Gene expression signatures for predicting prognosis of squamous cell and adenocarcinomas of the lung. Cancer Res. 2006;66(15):7466-7472.

38. Director's Challenge Consortium for the Molecular Classification of Lung Adenocarcinoma; Shedden K, Taylor JM, et al. Gene expressionbased survival prediction in lung adenocarcinoma: a multi-site, blinded validation study. Nat Med. 2008;14(8):822-827.

39. Beer DG, Kardia SL, Huang CC, et al. Gene-expression profiles predict survival of patients with lung adenocarcinoma. Nat Med. 2002;8(8): 816-824.

40. Liu Q, Li A, Tian Y, et al. The expression profile and clinic significance of the SIX family in non-small cell lung cancer. J Hematol Oncol. 2016; 9(1):119.

41. Tian Y, Liu Q, He X, et al. Emerging roles of Nrf2 signal in non-small cell lung cancer. J Hematol Oncol. 2016;9(1):14.

42. Sun W, Yuan X, Tian Y, et al. Non-invasive approaches to monitor EGFR-TKI treatment in non-small-cell lung cancer. J Hematol Oncol. 2015;8:95.

43. Guo K, Zhang ZP, Han L, et al. Detection of epidermal growth factor receptor mutation in plasma as a biomarker in Chinese patients with early-stage non-small cell lung cancer. Onco Targets Ther. 2015;8:3289-3296.

44. Xia QS, Xu J, Chen HY, et al. Association between an elevated level of HMGB1 and non-small-cell lung cancer: a meta-analysis and literature review. Onco Targets Ther. 2016;9:3917-3923.

45. Shen L, Wu X, Tan J, et al. Combined detection of dickkopf-1 subtype classification autoantibodies as biomarkers for the diagnosis and prognosis of non-small cell lung cancer. Onco Targets Ther. 2017;10:3545-3556. 
46. Arenberg DA, Keane MP, DiGiovine B, et al. Epithelial-neutrophil activating peptide (ENA-78) is an important angiogenic factor in nonsmall cell lung cancer. J Clin Invest. 1998;102(3):465-472.

47. Põld M, Zhu LX, Sharma S, et al. Cyclooxygenase-2-dependent expression of angiogenic CXC chemokines ENA-78/CXC Ligand (CXCL) 5 and interleukin-8/CXCL8 in human non-small cell lung cancer. Cancer Res. 2004;64(5):1853-1860.

48. Saintigny $\mathrm{P}$, Massarelli E, Lin S, et al. CXCR2 expression in tumor cells is a poor prognostic factor and promotes invasion and metastasis in lung adenocarcinoma. Cancer Res. 2013;73(2):571-582.

49. Shiels MS, Pfeiffer RM, Hildesheim A, et al. Circulating inflammation markers and prospective risk for lung cancer. J Natl Cancer Inst. 2013;105(24):1871-1880.

50. Spaks A. Role of CXC group chemokines in lung cancer development and progression. J Thorac Dis. 2017;9(Suppl 3):S164-S171.

51. Han N, Yuan X, Wu H, et al. DACH1 inhibits lung adenocarcinoma invasion and tumor growth by expressing CXCL5 signaling. Oncotarget. 2015;6(8):5877-5888.

52. Zhou SL, Zhou ZJ, Hu ZQ, et al. CXCR2/CXCL5 axis contributes to epithelial-mesenchymal transition of HCC cells through activating PI3K/ Akt/GSK-3ß/Snail signaling. Cancer Lett. 2015;358(2):124-135.

53. Lim JB, Chung HW. Serum ENA78/CXCL5, SDF-1/CXCL12, and their combinations as potential biomarkers for prediction of the presence and distant metastasis of primary gastric cancer. Cytokine. 2015;73(1): 16-22.
54. Zhang H, Xia W, Lu X, et al. A novel statistical prognostic score model that includes serum CXCL5 levels and clinical classification predicts risk of disease progression and survival of nasopharyngeal carcinoma patients. PLoS One. 2013;8(2):e57830.

55. Yanagawa J, Walser TC, Zhu LX, et al. Snail promotes CXCR2 ligand-dependent tumor progression in non-small cell lung carcinoma. Clin Cancer Res. 2009;15(22):6820-6829.

56. Kuo PL, Chen YH, Chen TC, Shen KH, Hsu YL. CXCL5/ENA78 increased cell migration and epithelial-to-mesenchymal transition of hormone-independent prostate cancer by early growth response-1/snail signaling pathway. J Cell Physiol. 2011;226(5):1224-1231.

57. Li A, King J, Moro A, et al. Overexpression of CXCL5 is associated with poor survival in patients with pancreatic cancer. Am J Pathol. 2011; 178(3):1340-1349.

58. Kawamura M, Toiyama Y, Tanaka K, et al. CXCL5, a promoter of cell proliferation, migration and invasion, is a novel serum prognostic marker in patients with colorectal cancer. Eur J Cancer. 2012;48(14): 2244-2251.

59. Xia J, Xu X, Huang P, He M, Wang X. The potential of CXCL5 as a target for liver cancer-what do we know so far? Expert Opin Ther Targets. 2015;19(2):141-146.

60. Heiskanen M. caArray_meyer-00191: Classification of human lung carcinomas by mRNA expression profiling reveals distinct adenocarcinoma subclasses. Available from: https:/www.ncbi.nlm.nih.gov/geo/ query/acc.cgi?acc=GSE83227. Accessed November 15, 2017. 


\section{Supplementary material}
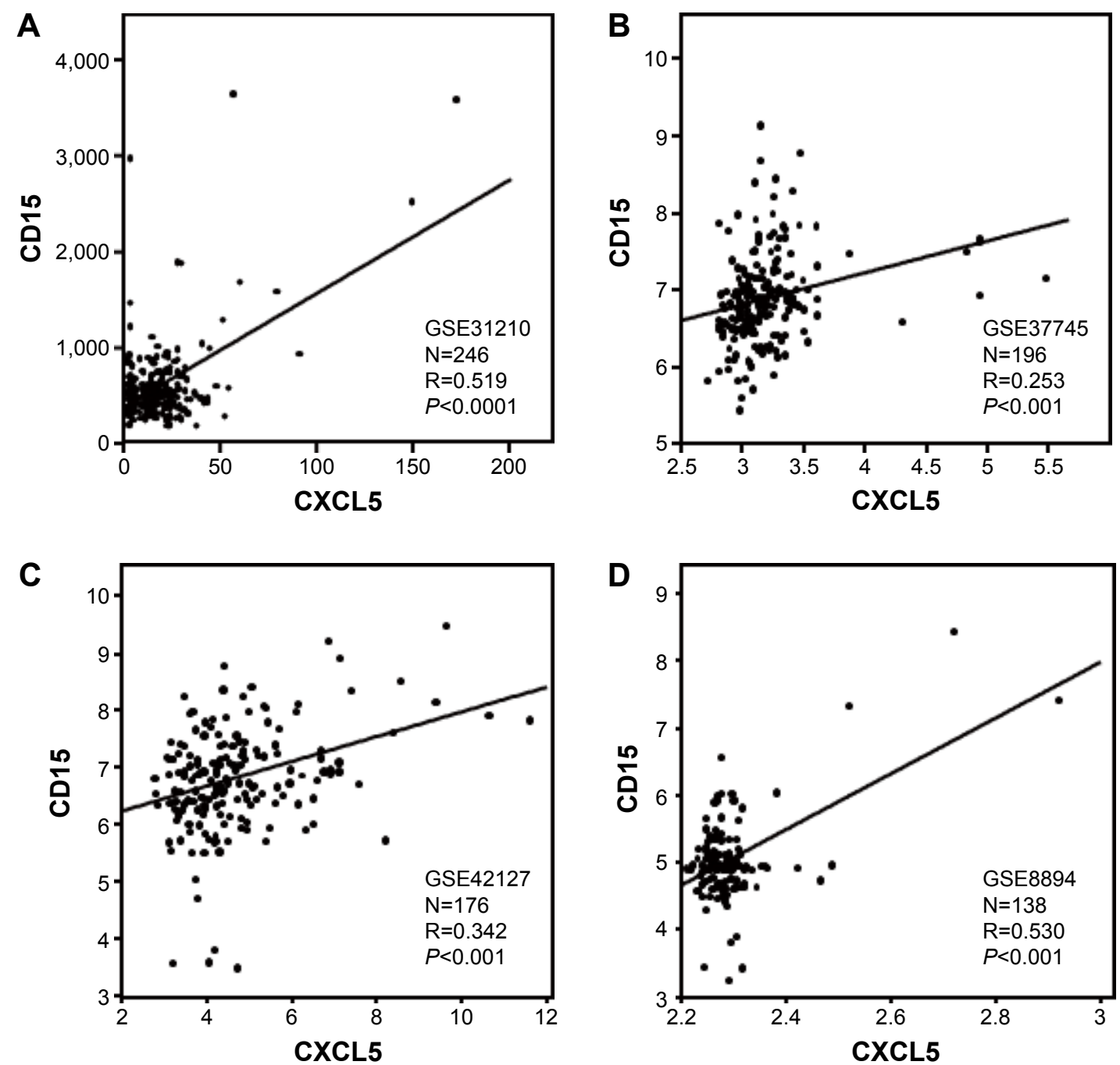

Figure SI Regression analysis between CXCL5 with neutrophil surface marker CDI5 in GSE31210 (A), GSE37745 (B), GSE42127 (C), and GSE8894 (D).

\section{Publish your work in this journal}

OncoTargets and Therapy is an international, peer-reviewed, open access journal focusing on the pathological basis of all cancers, potential targets for therapy and treatment protocols employed to improve the management of cancer patients. The journal also focuses on the impact of management programs and new therapeutic agents and protocols on patient perspectives such as quality of life, adherence and satisfaction. The manuscript management system is completely online and includes a very quick and fair peer-review system, which is all easy to use. Visit http://www.dovepress.com/testimonials.php to read real quotes from published authors. 